\title{
Los riesgos costeros: Retos para el desarrollo sostenible del turismo en los territorios insulares en el contexto del cambio climático.
}

Coastal risks: Challenges for the sustainable development of tourism in island territories in the context of climate change.

Dr.C Alexis Santiago Pérez Figueredo. ${ }^{1}$

\begin{abstract}
The achievement of sustainable tourism that integrates actions aimed at achieving the well-being of the tourist and the harmony of tourism development with its surroundings, taking as a scenario the preparation and confrontation before the impacts of climate change constitutes the fundamental challenge of tourism in the island territories.

The work is developed from the documentary and bibliographic review, the cartographic method and the modeling that allowed to design a procedure that contributes to the alignment in the management model of the tourist facilities of the implementation of the Life Task promoting the environmental performance and the perception of risk in the face of climate change.

As a result, the projections of the work are expressed and the study of the impacts of coastal risks is documented, basically the erosion and loss of the dunes, with proposals for actions that can be carried out for their prevention and coastal protection against processes of erosion promoting the development of risk management at the Carisol los Corales Hotel in the Cazonal Baconao coastal sector in the province of Santiago de Cuba.
\end{abstract}

Keywords: Sustainable Tourism, Climate Change, Life Task, Risk Perception

\section{Resumen}

La consecución de un turismo sostenible que integre acciones destinadas a lograr el bienestar del turista y la armonía del desarrollo turístico con su entorno, teniendo como escenario la preparación y el enfrentamiento ante los impactos del cambio climático constituye el reto fundamental del turismo en los territorios insulares.

\footnotetext{
${ }^{1}$ Universidad Oriente, alexis.figueredo@uo.edu.cu
} 
El trabajo se desarrolla a partir de la revisión documental y bibliográfica, el método cartográfico y la modelación que permitió diseñar un procedimiento que contribuye al alineamiento en el modelo de gestión de las instalaciones turísticas de la implementación de la Tarea Vida potenciando el desempeño ambiental y la percepción del riesgo ante el cambio climático.

Como resultados se expresan las proyecciones del trabajo y se documenta el estudio de los impactos por los riesgos costeros, en lo fundamental la erosión y perdida de las dunas, con propuestas de acciones que se pueden realizar para su prevención y la protección costera ante los procesos de erosión potenciando el desarrollo de la gestión de riesgos en el Hotel Carisol los Corales en el sector costero Cazonal Baconao en la provincia de Santiago de Cuba.

Palabras Clave: Turismo Sostenible, Cambio Climático, Tarea Vida, Percepción Del Riesgo

\section{Introducción}

El nuevo paradigma para el desarrollo de un turismo sostenible, constituye para el sector un doble reto, alcanzar una sostenibilidad de la calidad de sus servicios y atención al cliente, garantizando el bienestar del mismo y demostrar con su desempeño que el destino o producto turístico que se oferta, constituye un exponente de buenas prácticas hacia la protección y conservación del medio ambiente. (OMT, 2017).

El turismo constituye para el país una de las actividades económica que se debe convertir en uno de sus principales sectores para el desarrollo, donde se realizarán en los próximos años cuantiosas inversiones, ejemplo de ello son las que se han planteado para el 2030, un crecimiento en 252 nuevas instalaciones, 90 proyectos de incremento de categoría,13 proyectos de desarrollo inmobiliario asociados a campos de golf, 42 proyectos de recreación y más de 100 proyectos de logística ( Granma 5/8/2019). Estas inversiones estarán dirigidas en lo fundamental a potenciar la modalidad del turismo de sol y playa en la zona costera.

El cambio climático constituye una alta amenaza para los Estados Insulares por la alta vulnerabilidad de estos ante sus impactos. Entre las actividades económicas que serán afectadas, el turismo constituye una de las de mayor incidencia. Entre los impactos que se han identificado [(AMA, 2015), (CEPAL, 2018), (IPCC,2014)] generados por el cambio climático sobre el turismo se encuentran:

Desde el punto de vista económico:

- Reorientación de la modalidad de sol y playa como consecuencia de la elevación del nivel medio del mar y el retroceso de la línea de costa.

- Cambios en la preferencia gastronómica de los turistas por productos ecológicos como resultado al incremento de una cultura ambiental en el cliente ante el cambio climático.

- Cambio en los procesos de migración humana en las zonas costeras. 
Desde el punto de vista físico ambiental.

- Problemas de la biodiversidad y el stress de los ecosistemas marino costeros producto del cambio climático

- Incremento de la contaminación (por sólidos y plásticos) y la pérdida de la calidad ambiental del sitio turístico.

- Intensificación de los procesos de la erosión costera que potencian la pérdida de playas arenosas y dunas costeras.

- Aumento de la frecuencia y nivel de las inundaciones costeras y penetración del mar.

- Degradación del paisaje costero y fondos marinos.

En el Plan del Estado cubano para el enfrentamiento al cambio climático: Tarea Vida, (CITMA, 2017) se conceptúa como uno de sus objetivos la adopción de medidas para garantizar el desarrollo del turismo. En el análisis de sus cinco acciones estratégicas en cuatro de ellas existen indicaciones que deben tenerse en cuanta para el desarrollo del turismo y en las once tareas se evidencia una incidencia transversal al proceso de desarrollo de este sector.

Las actividades antrópicas desarrolladas en las zonas litorales pueden influir y aumentar el riesgo costero. Las posibles actividades humanas que pueden potenciarlos o están expuestas a estos son:

- El Desarrollo costero: incremento de la presión urbanística, infraestructuras y, en general, bienes y personas. puede suponer una presión excesiva sobre los recursos marinos y el litoral.

- Industria costera y puertos: las ventajas de la comunicación por mar han atraído a numerosas empresas. algunas de ellas son fuentes potenciales de vertidos o contaminación. también la ampliación de los puertos, para instalaciones económicas o de ocio, pueden invadir áreas de gran valor medioambiental y aislar sistemas naturales interdependientes.

- Agricultura: la agricultura intensiva puede reducir hábitat, disminuir la biodiversidad, reducir el caudal fluvial o subterráneo y sus residuos pueden modificar la composición del agua y su aporte al litoral. Los sedimentos y residuos provenientes de sustancias utilizadas en la agricultura pueden llegar al litoral originan importantes contaminaciones.

- Industria pesquera y acuicultura: la sobrepesca ha sido a menudo fuente de controversia, ciertas artes pesqueras y la acuicultura pueden alterar los hábitats.

- Actividades mar adentro: como extracción de petróleo y gas, extracción de áridos, minería de los fondos marinos o la instalación de parques eólicos marinos alteran no solo la línea de costa sino también el ecosistema costero, además pueden ser fuente de contaminación y vertidos.

- - Cambios en la calidad del agua: la gestión de residuos domésticos, de la actividad industrial o de los materiales arrastrados de las zonas agrícolas pueden 
afectar a la calidad del agua marina alterando las cualidades y características de las playas, marismas y humedales.

- Turismo y usos recreativos: aumenta la presión en las áreas de interés ecológico y puede suponer uso intensivo de recursos tan importante como el agua. Modifican no solo el valor escénico de los paisajes sino también pueden profundizar vulnerabilidades preexistentes o dar a origen a otras nuevas.

Las características del desarrollo turístico como actividad económica generan de por sí, vulnerabilidades tanto en el medio ambiente como en la sociedad, de no tomar las medidas adecuadas en su desarrollo se pueden profundizar impactos como:

- Las afectaciones originadas por malas prácticas en el manejo de los recursos naturales y paisajísticos de los destinos turísticos.

- En el ámbito social el déficit de servicios básicos que generan a su vez situaciones de estrés y presiones sobre los ecosistemas naturales con los que interactúan en el consumo y disponibilidad de recursos como el agua potable para la población, la calidad ambiental de los asentamientos humanos entre otros impactos.

Las zonas costeras por las características y fragilidad de sus ecosistemas y por constituir las áreas donde serán más evidentes los primeros impactos del cambio climático en los territorios insulares, requieren de una alta prioridad en la preparación y capacitación de los decisores y actores de los procesos de desarrollo para el enfrentamiento a estos posibles impactos.

Según estudios realizados por especialistas de la Universidad de Oriente y del CITMA así como, los resultados de tesis de grados, de diferentes programas de maestrías y doctorados, entre otros trabajos investigativos, existen vacíos e insuficiencias en la formación y conocimientos de la gestión de riesgos para el enfrentamiento al cambio climático en los tomadores de decisiones y actores del desarrollo en el territorio, por lo que se hace necesario potenciar su desarrollo hacia un proceso de cambio que permita contribuyendo a elevar la percepción del riesgo ante el cambio climático a través de:

- Fortalecimiento de los lazos existentes entre las dimensiones institucional, social y económica para potenciar la gestión de riesgos.

- Desarrollando capacidades para el enfrentamiento a los impactos del cambio climático desde el enfoque de la adaptación.

- Conocer los impactos del cambio climático a los que deben enfrentarse y cómo actuar para minimizar los impactos adversos.

- Gestionar las vulnerabilidades identificadas y asociadas a los impactos del cambio climático de forma efectiva.

- Identificar y aprovechar elementos que contribuyan a elevar la resiliencia entre los tomadores de decisiones y actores del desarrollo.

- Establecer prioridades y concertar esfuerzos en el desarrollo de medidas y acciones de adaptación con criterios claros teniendo en cuenta criterios de grupos vulnerables y enfoque de género. 
- Identificar los activos de adaptación, es decir, aquellas medidas ya adoptadas en el ámbito local que contribuyen actualmente a la adaptación.

- Crear sinergias entre medidas y acciones, así como superar los posibles costes de improvisación.

- Optimizar la asignación de los recursos disponibles frente al cambio climático y la adaptación.

- Fomentar la colaboración entre los sectores público y privado en materia de adaptación.

- Crear una cultura de la prevención común en materia de adaptación al cambio climático

Como parte de las indicaciones del Consejo de Estado para la implementación de la Tarea vida, en cada territorio los Organismo de la Administración Central del Estado (OACE), entre ellos el Ministerio de Turismo y sus instalaciones, deben elaborar un cronograma de trabajo que garanticen su implementación. De estas orientaciones emanan los principales intereses de coordinación en los territorios y ministerios.

Por indicaciones del Ministerio de Educación Superior las universidades deben crear grupos de expertos con la participación de profesores e investigadores y la conformación de un plan de acciones según necesidades y proyecciones del desarrollo territorial para garantizar la implementación de la Tarea Vida, con un orden de prioridad alto constituyendo este escenario, el marco de las relaciones entre los organismos, empresas y la universidad para la implementación de la Tarea Vida.

El papel de la universidad estará definido con el desarrollo del asesoramiento necesario para potenciar los estudios y la formación de capacidades para la gestión de riesgos ante los posibles impactos del CC, favoreciendo la percepción de riesgos y la participación de los trabajadores en la búsqueda de soluciones de los impactos generados por el cambio climático, proceso en el que se pueden desarrollar acciones de capacitación, evaluación de impactos y la realización de investigaciones relacionadas con este tema entre otras acciones.

Resultados del análisis de partes interesadas. Resumen

\begin{tabular}{llll}
\hline $\begin{array}{l}\text { Organizaciones } \\
\text { con intereses en } \\
\text { el proyecto. }\end{array}$ & $\begin{array}{l}\text { Interés y } \\
\text { actitud } \\
\text { hacia el } \\
\text { proyecto }\end{array}$ & $\begin{array}{l}\text { Papel en el } \\
\text { proceso de } \\
\text { preparación }\end{array}$ & $\begin{array}{l}\text { Participación } \\
\text { durante la } \\
\text { aplicación del } \\
\text { proyecto }\end{array}$ \\
\hline Gobiernos & Alto & Actor & $\begin{array}{l}\text { Tomador de } \\
\text { decisiones } \\
\text { Tomador de } \\
\text { decisiones- } \\
\text { actor }\end{array}$
\end{tabular}




\begin{tabular}{llll} 
Citma & Alto & Actor & $\begin{array}{l}\text { Tomador de } \\
\text { decisiones- } \\
\text { actor } \\
\text { Turismo }\end{array}$ Alto \\
& Actor & $\begin{array}{l}\text { Tomador de } \\
\text { decisiones- } \\
\text { actor }\end{array}$ \\
\hline
\end{tabular}

Análisis de causas y efecto de la situación problémica de la investigación

\begin{tabular}{|c|c|c|c|}
\hline Efecto & Problema & $\begin{array}{c}\text { Causa Limitadas acciones } \\
\text { de Formación }\end{array}$ & $\begin{array}{c}\text { Causa Débil } \\
\text { estrategia de } \\
\text { Comunicación } \\
\end{array}$ \\
\hline \multirow[t]{3}{*}{$\begin{array}{l}\text { Baja } \\
\text { percepción } \\
\text { del riesgo } \\
\text { ante los } \\
\text { impactos } \\
\text { generados } \\
\text { por el CC }\end{array}$} & $\begin{array}{l}\text { Limitado } \\
\text { conocimiento } \\
\text { sobre la } \\
\text { gestión de } \\
\text { riesgo ante los } \\
\text { impactos } \\
\text { generados por } \\
\text { el CC en el } \\
\text { territorio. }\end{array}$ & $\begin{array}{l}\text { Insuficiente } \\
\text { sobre la gestión de riesgos en } \\
\text { tomadores de decisiones y } \\
\text { actores del desarrollo. }\end{array}$ & $\begin{array}{l}1 . \text { a) } \\
\text { Insuficientes } \\
\text { estrategias de } \\
\text { bien público al } \\
\text { sobre el tema } \\
\text { en las } \\
\text { entidades y } \\
\text { gobiernos. }\end{array}$ \\
\hline & & $\begin{array}{l}\text { 1.a) Vacíos en los } \\
\text { programas de estudios de } \\
\text { temas sobre la gestión de } \\
\text { riesgos }\end{array}$ & $\begin{array}{l}\text { 2. Predominio } \\
\text { de un enfoque } \\
\text { reactivo en la } \\
\text { gestión de la } \\
\text { información y } \\
\text { la } \\
\text { comunicación } \\
\text { sobre la gestión } \\
\text { de riesgos }\end{array}$ \\
\hline & & $\begin{array}{l}\text { 2. Insuficientes ofertas de } \\
\text { cursos de capacitación sobre la } \\
\text { gestión de riesgo ante el CC. } \\
\text { 2.a) Desactualización de los } \\
\text { diagnósticos de las necesidades } \\
\text { de aprendizaje sobre este tema }\end{array}$ & \\
\hline
\end{tabular}

\section{Objetivo del trabajo}

Lo anteriormente argumentado demuestra la actualidad e importancia del trabajo encaminado a ¿Cómo potenciar desde las instalaciones del turismo la implementación de la tarea vida contribuyendo a las acciones dirigidas a la preparación y enfrentamiento ante los impactos del cambio climático en la zona costera donde se localiza esta instalación? 


\section{Metodología de trabajo}

\section{Métodos de trabajo.}

Entre los métodos de trabajo seleccionados se encuentran:

- Revisión documental y bibliográfica. Utilizado para determinar el estado del arte del marco teórico y la actualización del diagnóstico de la zona de estudio.

- Método matricial. Determinar los conflictos de uso _ uso y uso _ recurso que existen en la zona de estudio

- Método cartográfico, modelación. Modelación de los impactos del CC manifestado en la inundación costera según escenarios determinado.

- Observación, entrevistas y encuestas. Para conocer nivel de percepción del riesgo y actualización del diagnóstico.

\section{Resultados y discusión.}

La modelación del proyecto de trabajo se expresa a través del siguiente esquema que enfocado en la implementación de la Tarea vida en las instalaciones turísticas situadas en el sector costero seleccionado y contempla las etapas y fases de trabajo del estudio.

\section{IMPLEMENTACIÓN TAREA VIDA}

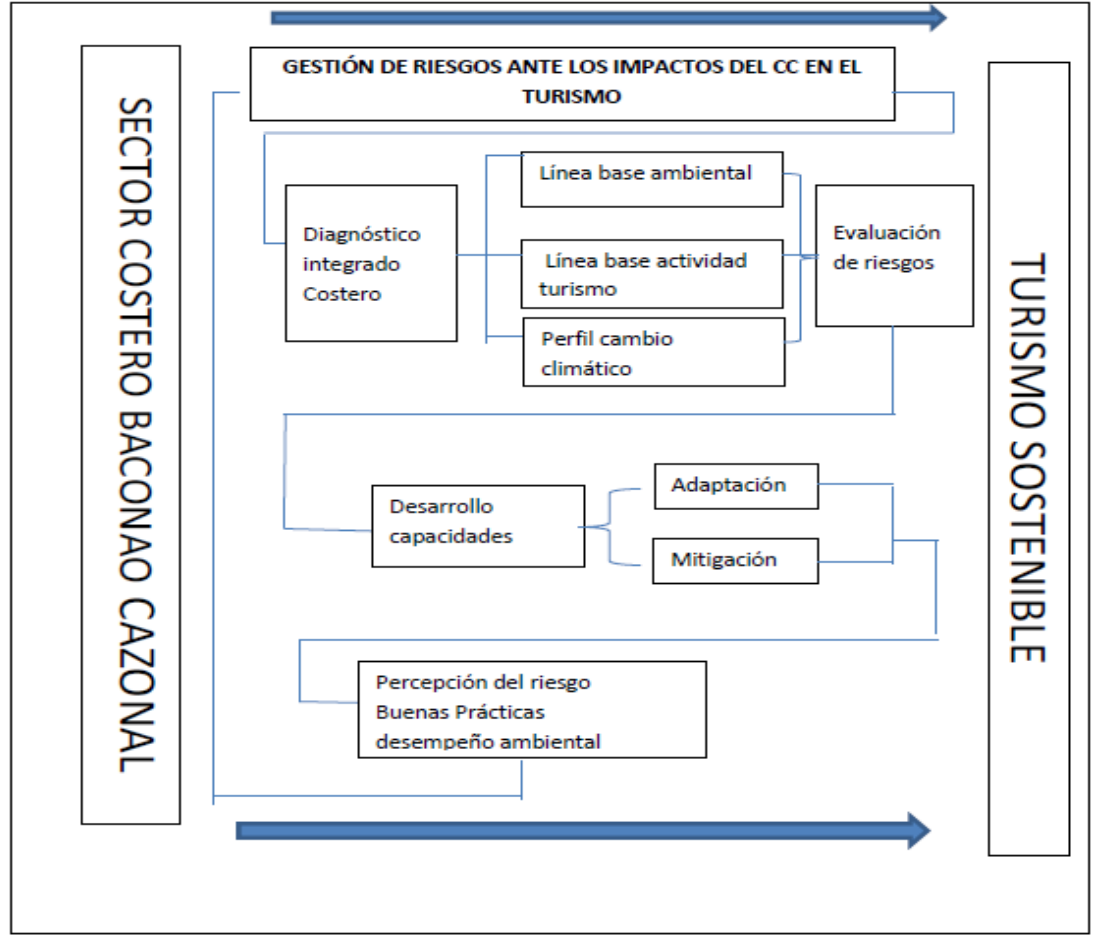

Del análisis del modelo anterior se deriva la organización del trabajo en dos etapas fundamentales: 
Evaluación de los riesgos

- Actualizacion del diagnóstico y la línea base

- Actualización de los estudios de PVR

- Estudio de percepción de riesgos

\section{Alineamiento estrategico. Formacion de capacidades}

- Alineamiento estratégico

- Formación de capacidades

- Desarrollo de buenas prácticas

\section{Los riesgos costeros}

Las actividades e infraestructuras económicas y sociales que están situadas en la línea de costa están expuestas al impacto de eventos y fenómenos naturales, a los que se deben añadir los producidos por los impactos derivados de las actividades humanas.

El riesgo se define [(Pérez 2014) (Molpeceres, Parra, Marambio, \& Ibáñez, 2012)] como la combinación de la probabilidad de ocurrencia de un suceso dado y de la magnitud de sus consecuencias. El riesgo considera la frecuencia con que se presentan ciertos estados o eventos y la magnitud de las consecuencias probables asociadas a la exposición a dichos estados o eventos de los bienes, personas, ecosistemas o actividades desarrolladas en la zona.

El cálculo del riesgo posee diversas formulaciones, pero todas ofrecen la evaluación cuantificada de los posibles daños y pérdidas derivadas de los impactos ocurridos cuando se hace tangible un peligro o amenaza. En sentido general el riesgo es directamente proporcional al peligro por la vulnerabilidad e inversamente proporcional a la capacidad de respuesta.

En la gestión de riesgos un concepto importante a considerar para la toma de decisiones, y que constituye el objetivo de la gestión de riesgos, es la vulnerabilidad, que está relacionada con las cualidades del entorno que lo hacen más susceptible ante un evento o fenómeno determinado ( por ejemplo las costas arenosas altas pueden ser más afectada en mayor magnitud que las costas arenosas bajas por la erosión eólica) y el nivel de exposición combinado con la capacidad de respuesta ante el impacto de las amenazas del territorio. En la medida que la evaluación de la vulnerabilidad sea conocida y exista el nivel de respuesta adecuado se pueden definir acciones que permitan minimizarla.

Otro concepto que ha venido tomando relevancia dentro de la gestión de riesgos es el de resiliencia definida como la capacidad de un sistema, comunidad o territorio expuesta a peligros para resistir, absorber, acomodarse y recuperarse de los efectos de un evento que la afecte (Pérez, 2018). Dentro de la gestión del riesgo costero la aplicación de este concepto puede viabilizar la práctica de los principios del manejo integrado costero.

Metodología de trabajo utilizando los criterios del manejo integrado de zonas costeras.

Proyección de los resultados a lograr: 


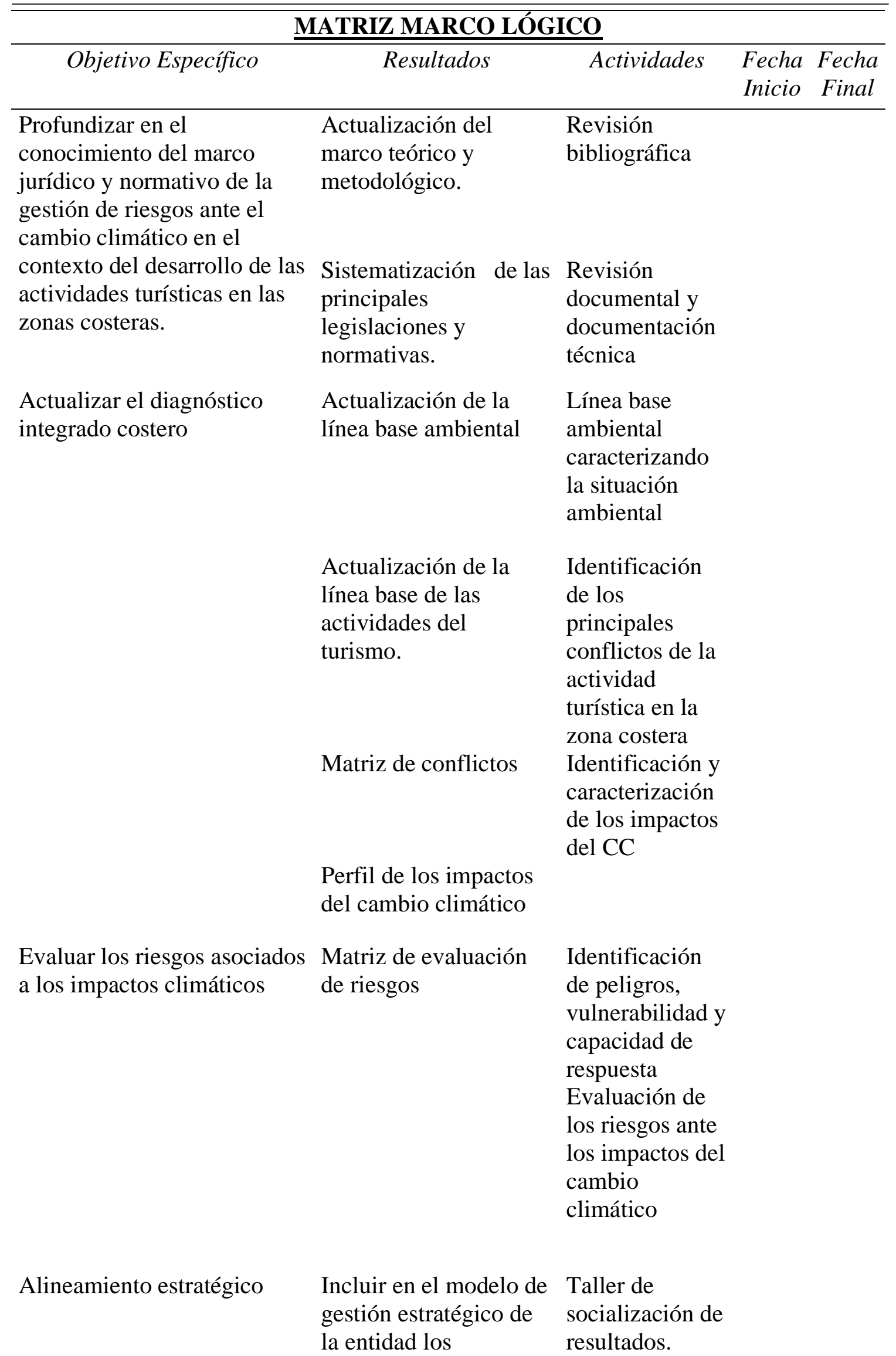


Potenciar la formación de capacidades para el enfrentamiento al CC

Evaluar los niveles de percepción de riesgos

Potenciar el desarrollo de buenas prácticas para el logro del turismo sostenible

Aplicar instrumentos para el control y evaluación elementos para la implementación de la tarea vida

Diagnóstico de las necesidades de capacitación

Diseño de diversas formas de capacitación para la formación de capacidades en el enfrentamiento al CC

Aplicación de instrumentos para investigar los niveles de percepción de riesgos

Diseño de buenas prácticas ambientales

Diseño de instrumentos y herramientas para el control y evaluación
Análisis y

definición de acciones con enfoque de adaptación Actualización del DNC

Propuestas de cursos, seminarios y talleres de capacitación enfocados en la adaptación

Evaluación de la percepción del riesgo ante los impactos del CC

Desarrollo de proyectos de buenas prácticas que constituyan evidencias del desempeñó ambiental de la institución

Proceso de evaluación y propuesta de plan de mejoras
TABLA INDICADORES

Resultado Línea Base

Indicador de monitoreo y seguimiento 


\begin{tabular}{ll}
\hline Evaluación del riesgo & $\begin{array}{l}\text { Actualización del marco } \\
\text { teórico metodológico } \\
\text { Actualización del diagnóstico } \\
\text { integrado costero } \\
\text { Actualización de los PVR }\end{array}$ \\
& \\
Formación de capacidades & $\begin{array}{l}\text { Actualización del DNC } \\
\text { para enfrentar los }\end{array}$ \\
impactos del CC & $\begin{array}{l}\text { Desarrollar acciones para el } \\
\text { control de la erosión costera y } \\
\text { la pérdida de dunas. } \\
\text { Diseño de cursos y de buenas } \\
\text { practicas }\end{array}$ \\
&
\end{tabular}

Presentación en eventos.

Realización talleres

Elaboración tesis

de grado y

Maestría

Elaboración de un

artículo

Desarrollo de cursos de capacitación y extensión, seminarios, talleres Elaboración de un articulo

\section{Indicadores de progreso}

Indicador clave o Línea de base Objetivo logrado indicador de cambio

1. Investigación

2. Capacitación

3. Extensión y alcance

4. Gestión
Línea base de los ecosistemas costeros desactualizada.

Estudios de PVR desactualizados

No existen cursos de capacitación ni de extensión
Actualización de las líneas base ambiental y del turismo.

Actualización de los estudios de PVR.

Desarrollo de un sistema de curso para la formación de capacidades para el enfrentamiento a los impactos del CC

No existen redes Se forma parte de una red ambiental para el de conocimiento turismo

No existe un plan de enfrentamiento al $\mathrm{CC}$
Implementación del Plan de enfrentamiento al CC: Tarea Vida. Desarrollo de acciones para el control de la erosión costera y la pérdida de dunas. 


\begin{tabular}{|c|c|c|}
\hline $\begin{array}{l}\text { 5. Desarrollo de } \\
\text { recursos humanos }\end{array}$ & $\begin{array}{l}\text { Existen vacíos } \\
\text { en el }\end{array}$ & $\begin{array}{l}\text { Se logra capacitar al } 90 \% \text { de los } \\
\text { trabajadores del hotel }\end{array}$ \\
\hline & $\begin{array}{l}\text { conocimiento } \\
\text { que tienen los } \\
\text { trabajadores } \\
\text { sobre el CC y es } \\
\text { bajo el nivel de } \\
\text { desempeño } \\
\text { ambiental de la } \\
\text { institución }\end{array}$ & $\begin{array}{l}\text { Es reconocida la institución por su } \\
\text { desempeño ambiental. }\end{array}$ \\
\hline
\end{tabular}

En una primera parte del trabajo y teniendo en cuenta las prioridades establecidas en el estudio de las playas turística se seleccionó la playa del Hotel Carisol los Corales para

Desarrollar un estudio preliminar con el interés de elevar la sensibilización ambiental de los tomadores de decisión y actores del desarrollo turístico ante esta problemática lo cual se logró, muestra de lo cual es el aval otorgado por el Grupo Cubanacan en el territorio para el desarrollo del trabajo.

Entre los riesgos costeros que han sido caracterizados en el sector están:

- La penetración e inundación costera,

- la pérdida de la duna,

- la erosión costera y

- blanqueamiento de corales (este último en el frente de playa Cazonalito),

Todos ellos combinado con la magnitud de los impactos del cambio climático.

La modelación que ese expone en la siguiente figura sobre el comportamiento de la inundación costera en el sector de estudio, en un escenario de aumento de la temperatura a dos grados centígrados se puede apreciar la magnitud del impacto, lo que justifica la importancia que tiene la adopción de medidas preventivas dirigida a minimizar vulnerabilidades ante este riesgo.

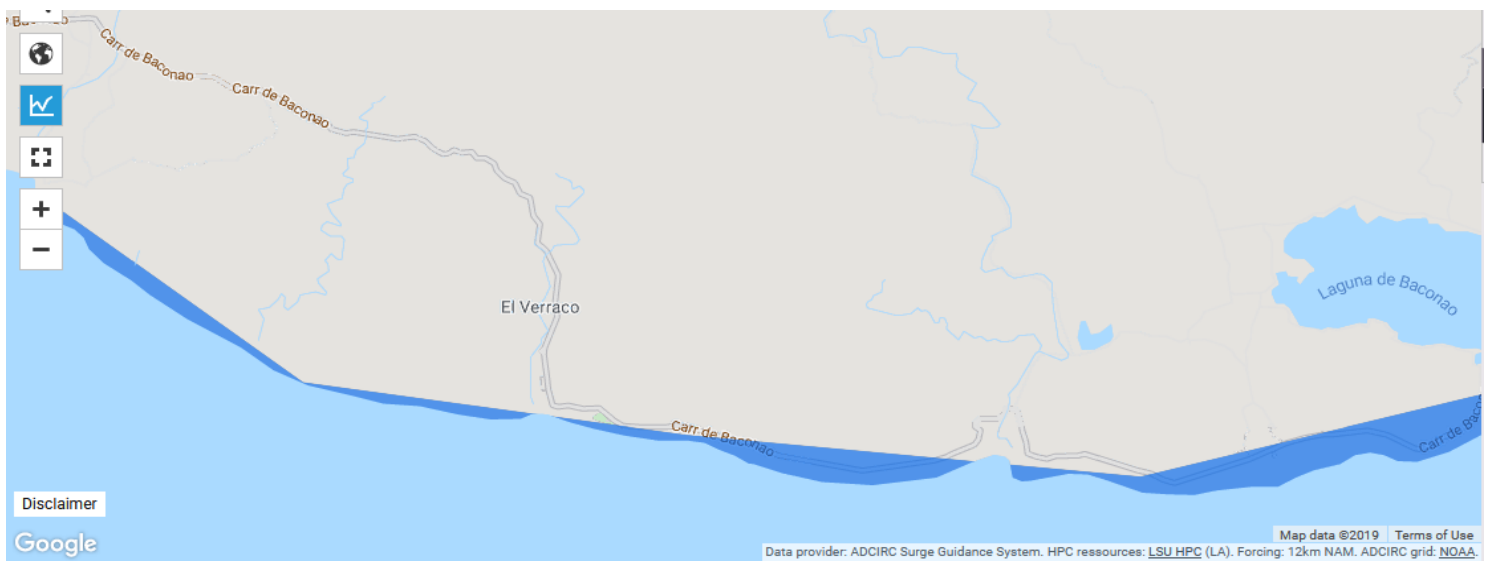

Las playas en el sector no tienen características óptimas para la explotación turística dado lo limitado del área de sol y los procesos erosivos que la afecta, así como por el grosor de la arena que en ocasiones es constituida por una grava de grano fino a medio. Solamente 
la sección correspondiente a la zona de baño del hotel posee condiciones adecuadas para el desarrollo de la modalidad de sol y playa.

La relación que se establece con otras playas en sus alrededores, donde existen evidencias de deterioro ambiental que inciden en la calidad paisajística del entorno, hacen que las acciones por prevenir la erosión costera se consideren como una prioridad dentro de los estudios de PVR que se realicen en la zona por lo que se hace necesario elaborar un proyecto de restauración física de la duna como primera barrera de protección de la playa.

La gestión de los sistemas duna - playa abarcan un amplio conjunto de medidas. Responden a criterios de conservación ambiental y de desarrollo económico sostenible por lo que los estudios e investigación de los procesos naturales litorales y para mantener, rehabilitar, restablecer la cubierta vegetal, proteger, regular y zonificar las actividades humanas constituyen acciones necesarias, a considerar y que han tenido resultados satisfactorios en otras playas.

El desarrollo de la actividad turística en estos sectores impone la introducción de instrumentos de gestión ambiental, que permitan asegurar la integración de los recursos turísticos con el medio natural, social y cultural del destino, con énfasis en el frente de playa de su sector hotelero.

En el sistema duna-playa, se realiza un constante intercambio dinámico de arena y son interdependientes entre ambos elementos esto provoca que las alteraciones en las playas arenosas afecten a las dunas costeras y viceversa. En consecuencia, para que el manejo de este ecosistema sea efectivo, se debe considerar su funcionamiento de forma integral, pues son altamente dinámicos, dependen del transporte de sedimentos por el viento y en menor grado, del efecto combinado de la marea de tormenta, la marea astronómica y el oleaje, por lo tanto, son vulnerables a las variaciones de cualquiera de estos elementos o procesos

Las dunas costeras, forman junto a las barras submarinas, los elementos más importantes dentro de la morfología de una playa, de hecho, constituyen sus reservas de arena, que son movilizadas para lograr minimizar el efecto erosivo de las olas en momentos de eventos extremos, acomodando el perfil transversal a las condiciones más severas de la energía del oleaje. Esta función protectora de las dunas litorales adquiere más importancia, ante los actuales desafíos del cambio climático.

Los procesos naturales y las actividades humanas que se desarrollan en estos sistemas pueden alterar el equilibrio dinámico que los caracteriza. Con frecuencia, el efecto de estos cambios no es visible de inmediato, por lo que las consecuencias de modificar o interrumpir el flujo del viento, del agua, o el aporte de los sedimentos no son visibles hasta que fenómenos hidrometeorológicos extremos y/o geotectónicos ponen a prueba la resiliencia y resistencia de estos sistemas.

\section{Pasos básicos para la rehabilitación del sistema duna - playa}

Antes que cualquiera de las fases de la rehabilitación, es importante conocer la dinámica de la costa y determinar si existe una fuente de arena disponible para la reconstrucción de 
dunas, ya que, si no hay arena, no hay playa ni duna posible. A grandes rasgos, la rehabilitación de sistemas dunares se consigue a través de seis pasos básicos (Ley-Vega et al., 2007).

1. Eliminación o control de las fuentes de la degradación.

2. Recuperación topográfica.

3. Recuperación de la vegetación (cuando es necesario).

4. Protección del sitio, establecimiento de adecuados accesos.

5. Divulgación.

6. Seguimiento a largo plazo mediante monitoreo

\section{Técnicas a utilizar}

Existen diversas técnicas que se pueden utilizar entre ellas los captadores pasivos de arenas, los recubrimientos y mulches así como la reposición de arenas a través de medios técnicos y maquinarias

Los captadores pasivos de arenas constituyen los medios menos costosos y agresivos con el medio ambiente por lo que su uso es más extendido, con el empleo de esta técnica se logra reconstruir la topografía del cordón dunar y llevarla hasta alcanzar una morfología lo más parecida a la que existía primitivamente. Su función es reducir la velocidad del viento por fricción y con ello, disminuir la carga de arena transportada, propiciando la acumulación de arena, aumentando la altura y anchura del depósito Estos sistemas contrarrestan la erosión eólica y aportan una mayor estabilidad al depósito arenoso

Existen dos tipos de captadores, de acuerdo con su emplazamiento y los objetivos perseguidos. Los sistemas de captadores estructurales para las zonas donde no existe vegetación y el cordón dunar está prácticamente ausente, otro tipo lo son los sistemas de captadores de apoyo a las plantaciones, para las zonas donde existe algo de vegetación y el cordón dunar mantiene todavía su estructura

\section{Conclusiones}

- El cambio climático constituye una realidad que por su magnitud afectará a toda la sociedad en su conjunto. Para el turismo como actividad económica esto puede implicar un cambio y reorientación de modalidades para la práctica de las actividades turísticas y en la perdida de la calidad ambiental de los sitios turísticos, con el deterioro de valores escénicos entre otros impactos. Estos impactos serán mayores en las zonas marino costeras donde además se pueden acrecentar riesgos costeros como la erosión del sistema playa duna y la inundación costera entre otros.

- Esta situación connota la importancia que tiene el trabajo preventivo que se debe desarrollar y que constituye el objetivo de la implementación de la Tarea Vida para las instalaciones turísticas.

- La sistematización realizada como resultados de la revisión bibliográfica y documental, y de los informes técnicos e investigaciones realizadas, permitió profundizar y actualizar conocimientos relacionados con el MIZC y validar la 
connotación e importancia que tienen los principios del manejo integrado de la zona costera como recursos teóricos metodológicos para la gestión de los riesgos costeros.

- Concebir la gestión de riesgos costeros desde las perspectivas del Decálogo del MIZC contribuye a potenciar su integración alineada con los instrumentos del modelo estratégico de las instituciones e incorporar las visiones de la distinta legislación y normativa que sobre la gestión de la zona costera generando acciones que tributan al logro de la sostenibilidad de estos territorios.

\section{Referencias bibliográficas}

AMA, (2015). Memoria de estudio de peligros, vulnerabilidad y riesgos (PVR). Editorial Academia, Cuba

Cepal, (2018). Impactos del Cambio Climático en América Latina y el Caribe. UN, N Y

Citma, (2017). Plan de Estado de la República de Cuba para el enfrentamiento al cambio climático:

Tarea Vida. Editorial Academia, Cuba

Febles, G. (2009). La diversidad biológica en Cuba, características y situación actual. Estrategia nacional y plan de acción. Revista Cubana de Ciencia Agrícola, Tomo 43, Número 3, 2009

Granma, (2019). Proyecciones del desarrollo del turismo en Cuba hasta el 2030, Periódico Granma 5/8/2019

Hernández, P. (2012). Geografía de Cuba 9no grado. Editorial Pueblo y Educación. La Habana. Cuba. ISBN 978-959-13-2159-6

IPCC, (2014). IV Informe del panel intergubernamental del Cambio Climático, UN. NY

Ley-Vega. (2007). Manual de restauración de dunas costeras. Dirección General de Costas. España: Editorial. Ministerio del Medio Ambiente

López, A. (2018). Manejo integrado de zonas costeras y planificación espacial marina: Contexto y conceptos. Curso Manejo integrado de zonas costeras - Planificación espacial marina - 2018 13-17 agosto 2018, Santa Marta, Colombia

OMT, (2017). Declaración de turismo sostenible, Organización Mundial de Turismo. UN, NY

Pérez, E (2017). Tarea de vida, Entrevista Periódico Juventud Rebelde, Cubadebate 10 de junio del 2017

RED Atlántica para la Gestión de Riesgos Costeros. Riesgos costeros: cómo reconocerlos y enfrentarse a ellos, (SF) 


\section{PARA CITAR EL ARTÍCULO INDEXADO.}

Pérez Figueredo, A. S. (2021). Los riesgos costeros: Retos para el desarrollo sostenible del turismo en los territorios insulares en el contexto del cambio climático. Explorador Digital, 5(1), 317-333. https://doi.org/10.33262/exploradordigital.v5i1.1505 


\section{Ciencia \\ Ligital}

El artículo que se publica es de exclusiva responsabilidad de los autores y no necesariamente reflejan el pensamiento de la Revista Explorador Digital.

El artículo queda en propiedad de la revista y, por tanto, su publicación parcial y/o total en otro medio tiene que ser autorizado por el director de la Revista Explorador Digital.
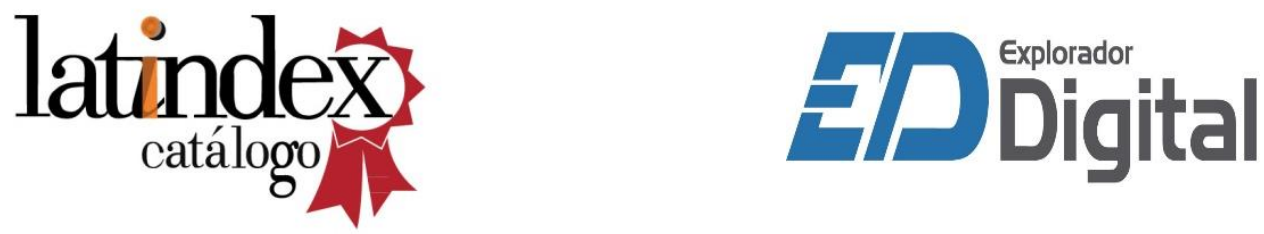